\section{REVOLUTIONARY BIOCHEMISTRY}

Help patients prevent oral diseases with a solution that is clinically proven to reduce bacterial plaque. Citrox has been innovatively developed as a key ingredient in a wide range of dental products, including mouth rinse.

Citrox is created using the bioflavonoids in the piths and pulp of dried, ground bitter oranges. This fine powder is mixed with other natural substances to produce a powerful anti-microbial, anti-oxidant, and anti-inflammatory agent. A concentration of just $0.25 \%$ Citrox solution can eliminate biofilm cells in under 10 minutes.

With the ability to kill 99.9998\% of bacteria, viruses, and fungi, Citrox proves itself as a highly effective natural disinfectant - ideal for dental applications. For more information please call 01480 862084, email info@curaprox.co.uk.

\title{
STAYING CURRENT IN A TECHNICAL REVOLUTION
}

Staying abreast of the evolution of digital dentistry can be challenging but Solvay Dental $360^{\text {ix }}$ is offering a unique opportunity for dental professionals. The Solvay Dental $360^{\text {Tsx }}$ team can visit your dental practice and deliver professional Lunch and Learn sessions. They will explain the digital workflow for the fabrication of removable partial dentures (RPDs) and introduce you to Ultaire ${ }^{\mathrm{rx}}$ AKP.

Ultaire $^{\mathrm{ma}} \mathrm{AKP}$ is a new high performance polymer, custom developed specifically for removable partial dentures and offers a biocompatible, metal-free option that provides stable retention, increased comfort and superior aesthetics.

Graham Bryant, Clinical Dental Technician (CDT) at the Natural Denture Clinic in Hampshire uses Ultaire ${ }^{\mathrm{Tw}}$ AKP to create lightweight, metal-free removable denture (RPD) frames. This material is strong but highly compliant and fits seamlessly into the digital workflow.
'I have completed several RPD cases using Ultaire $^{\mathrm{Tn} \mathrm{TS}}$ AKP and I really like working with it.' said Graham. 'It is great to have an alternative to monomer-based acrylic or chrome that is also biocompatible and taste-free. It enables me to use digital technology and provide accurate, retentive RPDs for improved comfort and fit, and my patients are delighted with the natural feel and look of Ultaire ${ }^{\text {mx }}$ AKP too.'
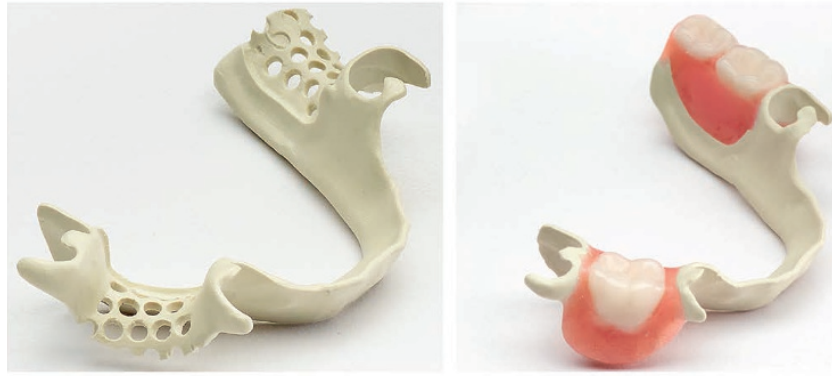

To book a Solvay Dental $360^{\text {mit }}$ Professional Lunch and Learn or to find more information Ultaire $^{\text {Twx }}$ AKP and Dentivera ${ }^{\text {Tw }}$ milling discs, please visit www.solvaydental360.com.

\section{CHART THE WAY}

The intuitive Florida Probe can be used to complete a comprehensive periodontal examination in less than 10 minutes. This computerised probe provides consistent precision $(0.2 \mathrm{~mm})$ and force $(15 \mathrm{~g})$, enabling practitioners to achieve highly accurate results during periodontal examinations.

Offering an efficient way for practitioners to chart their patients' periodontal health, the Florida Probe can be purchased either as the traditional kit or, alternatively, as the innovative Voice Works system, which facilitates voice-activated charting.

Either way, the operator can work alone using the same Florida Probe software, including its risk assessment feature and excellent handouts, which are really useful for patient communication. For more information, call Clark Dental on 01268733 146, email info@clarkdental.co.uk or visitwww. clarkdental.co.uk.

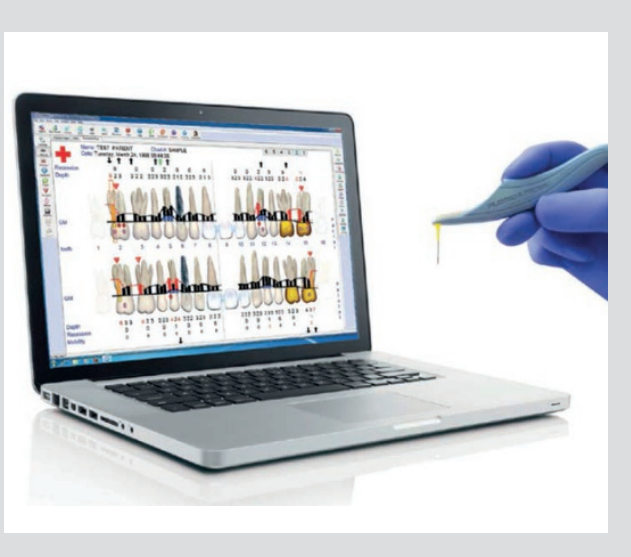

\section{THE MOST SOPHISTICATED SECRETARY}

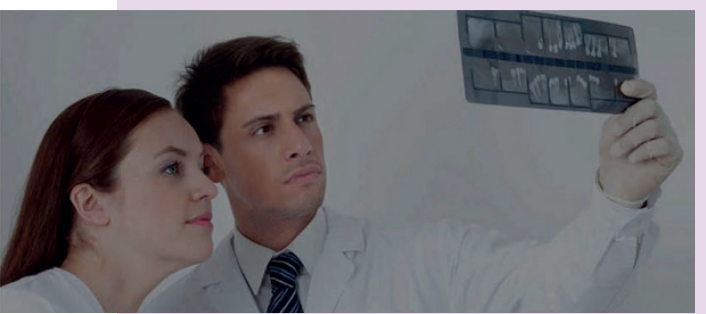

Do you want to save time reporting on radiographs but still have all of the essential information to create the best possible care plan? Storage solution PROPACS from
PRO Diagnostics UK can help.

Radiographs uploaded to PROPACS can be sent to a team of specialist dental radiologists for expert appraisal. Full reports will be sent back to your account within a 24 hour period, quickly giving you the information you need to create the best possible care plan for the patient.

Furthermore, as these images are stored directly into your PROPACS account you can share them with authorised colleagues or technicians in order to facilitate treatment and the creation of any restorative devices. All they need to do is request free log-in details and they can access the images. The whole process is GDPR compliant and significantly reduces the chances of patient information being leaked.

Available to access anywhere, any time, PROPACS is the ultimate sophisticated secretary and will streamline your diagnostic procedures like never before. For more information, please visit www. prodentalradiology.com or email sales@ prodiagnostics.co.uk. 